\title{
The Effects of Extravehicular Activity (EVA) Glove Pressure on Hand Strength
}

\author{
Miranda Mesloh \\ Lockheed Martin \\ Johnson Space Center, Houston, TX \\ Scott England \\ MEI Technologies, Inc. \\ Elizabeth Benson \\ MEI Technologies, Inc. \\ Shelby Thompson \\ Lockheed Martin \\ Sudhakar Rajulu \\ National Aeronautics and Space Administration
}

\section{ABSTRACT}

The purpose of this study was to characterize hand strength, while wearing a Phase VI Extravehicular Activity (EVA) glove in an Extravehicular Mobility Unit (EMU) suit. Three types of data were collected: hand grip, lateral pinch, and pulp-2 pinch, under three different conditions: bare-handed, gloved with no Thermal Micrometeoroid Garment (TMG), and glove with TMG. In addition, during the gloved conditions, subjects were tested when unpressurized and pressurized (4.3 psi).

As a percentage of bare-hand strength, the TMG condition showed reduction in grip strength to $55 \%$ unpressurized and $46 \%$ pressurized. Without the TMG, grip strength increased to $66 \%$ unpressurized and $58 \%$ pressurized of barehand strength. For lateral pinch strength, the reduction in strength was the same for 
both pressure conditions and with and without the TMG, about $85 \%$ of bare-hand. Pulp-2 pinch strength with no TMG showed an increase to $122 \%$ unpressurized and $115 \%$ pressurized of bare-hand strength. While wearing the TMG, pulp-2 pinch strength was $115 \%$ of bare-hand strength for both pressure conditions.

Keywords: EVA, Spacesuit, Hand Strength, Glove, Pressurized Suit, Phase VI Glove, NASA, Human System Integration, Grip, Pinch

\section{INTRODUCTION}

Proper human system integration is essential to mitigate risks to mission and crew while promoting human health and performance. This study primarily focused on EVA glove performance, in terms of hand and finger strength, in order to generate requirements for the next generation glove and the design of hardware (e.g., tools, controls, mobility aids, hatch operation, etc.). In fact, a vital step in human-centered design is the application of these results to designing better human-machine interfaces.

The hand and finger strength results reported are part of a large set of data collected to characterize the Phase VI EVA glove. All data was collected by the Habitability and Human Factors Branch at NASA's Johnson Space Center. Other data collected included: hand mobility, tactility, dexterity, and functional task performance using EVA tools and a cursor control device. Due to the immense amount of data collected, this publication will focus only on the hand strength results.

The specific hand strength postures (grip, lateral pinch, and pulp-2 pinch) were chosen because of the availability of values in existing literature for comparison reasons and the popularity of their use when manipulating EVA tools. Current NASA requirements state that the next generation EVA glove must perform as good, or better, than the most current Phase VI glove. Therefore, it was important that quantifiable and replicable measures be collected to characterize performance. To that end, a set of conditions that generalized known operations was developed.

\section{METHODOLOGY}

The included results were compiled from 8 subjects (4 males and 4 females) that represented a range of hand anthropometry. Subjects were instructed to exert and sustain a maximum grip or pinch force for a total of 3 seconds during each trial. The force data was recorded using a data acquisition system from which the maximum produced force was extracted. This was repeated until a total of three trials were completed. A period of 2 minutes of rest was given between trials to prevent fatigue (Rajulu, 1993). The test conductor then verified that the maximum strength values for each trial were within $10 \%$ of each other. This was done to ensure the accuracy of results. If a trial was outside of this $10 \%$ interval, the value was excluded and an additional trial was performed. The three trials were then averaged to produce a 
final strength value for that particular subject and condition. This procedure was implemented for all types of hand strength and conditions involved in the test. Figure 1 shows the glove without a TMG. Figure 2 shows the test set-up used for each subject in the EMU supported by a suit stand.
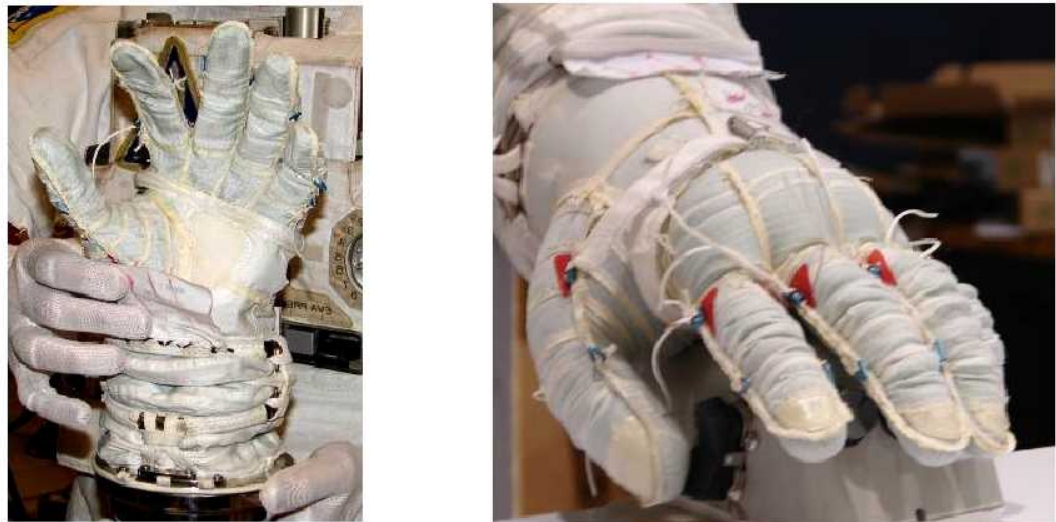

Figure 1. Phase VI EVA Glove with no TMG shown unpressurized (left) and pressurized (right).

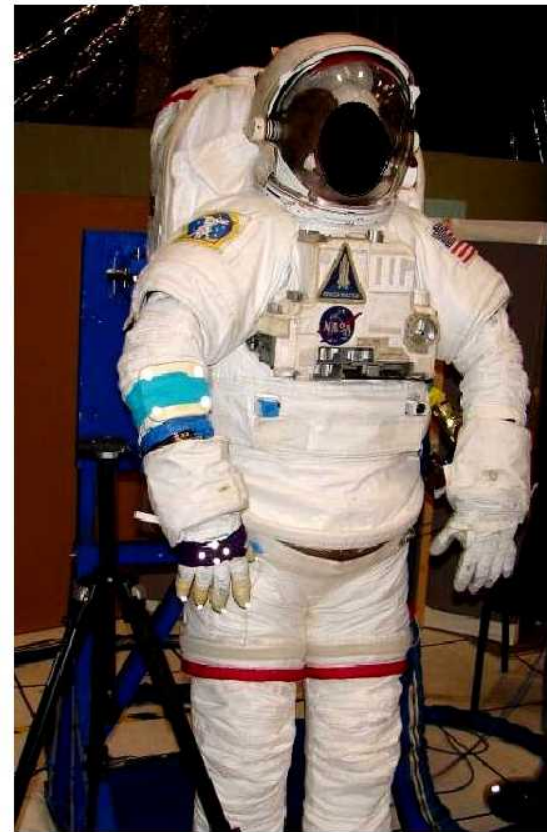

Figure 2. Subject test set-up in Extravehicular Mobility Unit (EMU) supported by suit stand with TMG

Results will be discussed in terms of actual force applied (pound-force, lbf) and as a percentage of bare-hand strength. The latter is important in characterizing the glove itself instead of the strength capabilities of the test subjects. It should be 
noted that there was no correlation found between subject strength and bare-hand strength percentage.

Grip strength was measured using a JAMAR hand dynamometer. Pinch strength was measured using a calibrated load cell. When collecting the strength data, subjects stood using a similar arm posture seen when wearing the suit. This was done as an effort to eliminate as many variables as possible.

\section{RESULTS}

\section{HAND GRIP STRENGTH}

The hand posture used while gripping the dynamometer is seen in Figure 3 and the average, minimum, and maximum force is shown in Table 1 . As a percentage of bare-hand strength, the TMG condition showed reduction in grip strength to $55 \%$ unpressurized and $46 \%$ pressurized. Without the TMG, grip strength increased to $66 \%$ unpressurized and $58 \%$ pressurized of bare-hand strength. In terms of the interaction, grip strength was reduced the most when wearing a TMG at $4.3 \mathrm{psi}$ and least with no TMG and unpressurized. The percentage grip strength for no TMG at 4.3 psi was similar to that found for with TMG and no pressure (see Figure 4).
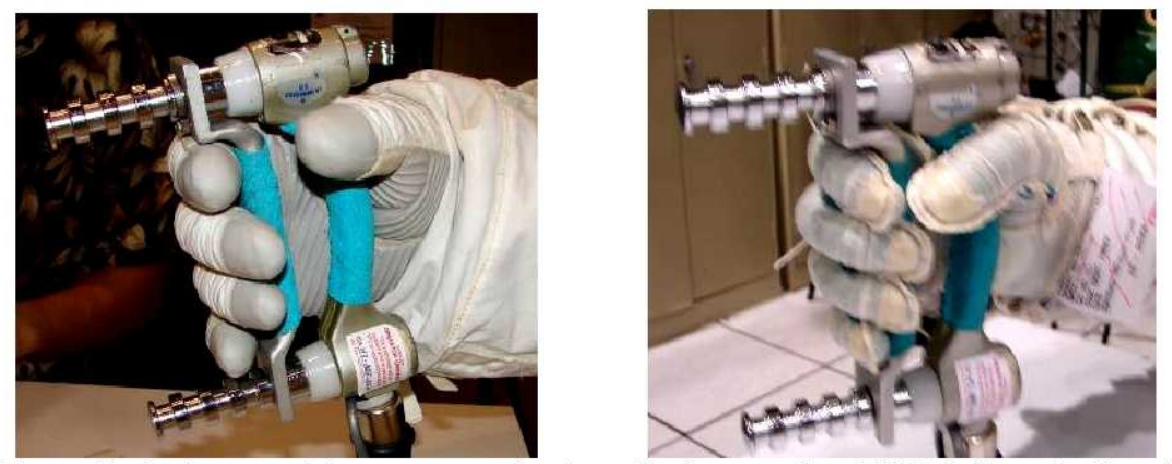

Figure 3. Posture used to measure grip strength shown with a TMG (left) and without a TMG (right). 
Table 1. Grip Strength Results

\begin{tabular}{|c|c|c|c|c|c|}
\hline & \multirow[b]{2}{*}{$\begin{array}{l}\text { Bare- } \\
\text { Hand }\end{array}$} & \multicolumn{2}{|c|}{ Gloved (No TMG) } & \multicolumn{2}{|c|}{ Gloved (TMG) } \\
\hline & & 0 psi & $4.3 \mathrm{psi}$ & 0 psi & $4.3 \mathrm{psi}$ \\
\hline Minimum & 60 & 43 & 35 & 33 & 24 \\
\hline Maximum & 135 & 80 & 66 & 73 & 66 \\
\hline Mean & 93 & 59 & 52 & 50 & 43 \\
\hline
\end{tabular}

Note. All values are reported as pound-force (lbf).

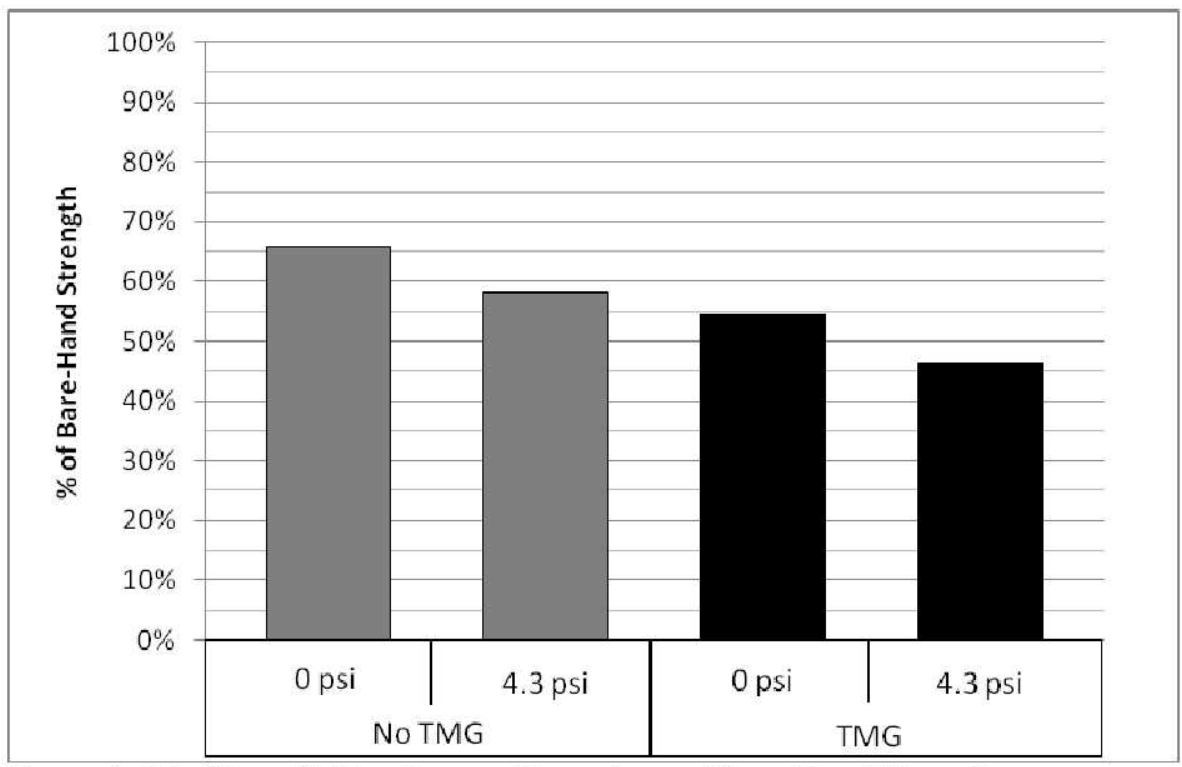

Figure 4. Grip Strength Results as a Percentage of Bare-Hand Strength

These results suggest that overall the TMG has a greater affect at both psi conditions on reducing grip strength as shown by lower percentages as compared to no TMG.

\section{PINCH STRENGTH}

The hand posture used while gripping the load cell can be seen in Figure 5. Lateral pinch posture is also commonly referred to as a key pinch as it resembles the hand configuration used when starting a car or unlocking a door (Mathiowetz, 1984). The load cell was positioned between the pad of the thumb and the second knuckle of the bent index finger. The pulp- 2 pinch hand posture seen in Figure 5 utilizes the 
thumb and index finger. While keeping the fingers as straight as possible, the subject used the pads of the thumb and index finger to apply a force to the load cell.
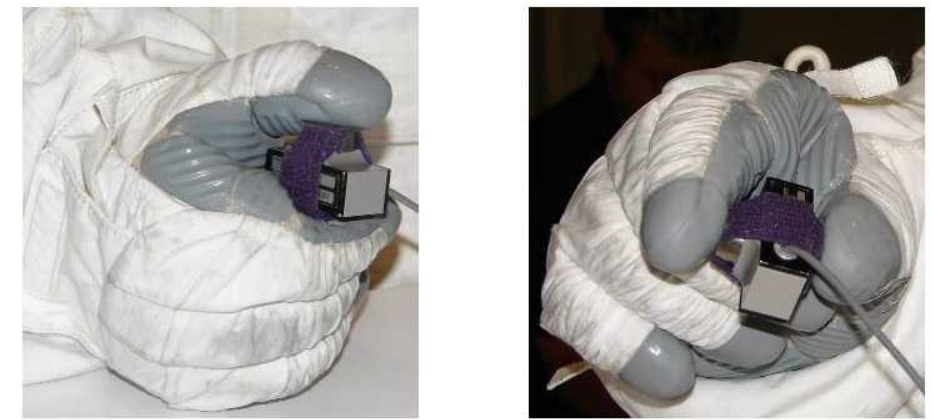

Figure 5. Posture used to measure lateral pinch strength (left) and pulp-2 pinch strength (right).

\section{Lateral Pinch}

Table 2 shows the average, minimum, and maximum lateral pinch strength values. As a percentage of bare-hand strength, the TMG condition showed reduction in lateral pinch strength to $84 \%$ unpressurized and $86 \%$ pressurized. Without the TMG, lateral pinch strength was $83 \%$ unpressurized and $84 \%$ pressurized of barehand strength.

Table 2. Lateral Pinch Strength Results

\begin{tabular}{lccccccc}
\hline & & \multicolumn{2}{c}{ Gloved (No TMG) } & & \multicolumn{2}{c}{ Gloved (TMG) } \\
\cline { 3 - 4 } \cline { 6 - 7 } & $\begin{array}{l}\text { Bare- } \\
\text { Hand }\end{array}$ & 0 psi & 4.3 psi & & 0 psi & 4.3 psi \\
\hline Minimum & 17 & 14 & 13 & & 13 & 8 \\
Maximum & 28 & 24 & 23 & & 26 & 22 \\
Mean & 21 & 20 & 16 & & 19 & 16 \\
\hline
\end{tabular}

Note. All values are reported as pound-force (lbf). 


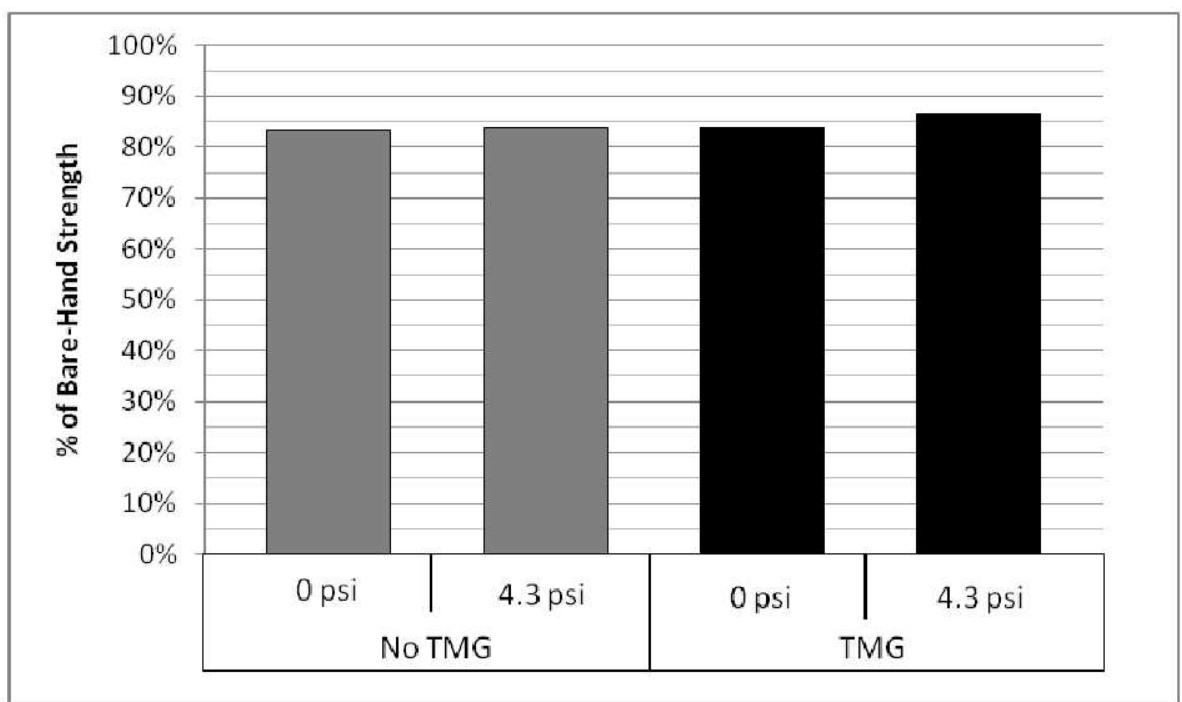

Figure 6. Lateral Pinch Strength Results as a Percentage of Bare-Hand Strength

Overall, lateral pinch strength was reduced by about $15 \%$ compared to bare-hand strength. Pressurizing the glove actually provided a slight increase in performance for both TMG and non-TMG cases. Further explanation will be provided in the discussion.

\section{Pulp-2 Pinch}

Table 3 shows the average, minimum, and maximum pulp-2 pinch strength values. As a percentage of bare-hand strength, the TMG condition showed an increase in pulp-2 pinch strength to $115 \%$ for both unpressurized and pressurized. Without the TMG, pulp-2 pinch strength was $122 \%$ unpressurized and $115 \%$ pressurized of bare-hand strength.

Table 3. Pulp-2 Pinch Strength Results

\begin{tabular}{lcccccc}
\hline & & \multicolumn{2}{c}{ Gloved (No TMG) } & & \multicolumn{2}{c}{ Gloved (TMG) } \\
\cline { 3 - 4 } \cline { 6 - 7 } & $\begin{array}{c}\text { Bare- } \\
\text { Hand }\end{array}$ & 0 psi & 4.3 psi & & 0 psi & 4.3 psi \\
\hline Minimum & 7 & 9 & 9 & & 7 & 9 \\
Maximum & 18 & 19 & 19 & & 18 & 17 \\
Mean & 12 & 12 & 12 & & 13 & 12 \\
\hline
\end{tabular}

Note. All values are reported as pound-force (lbf). 


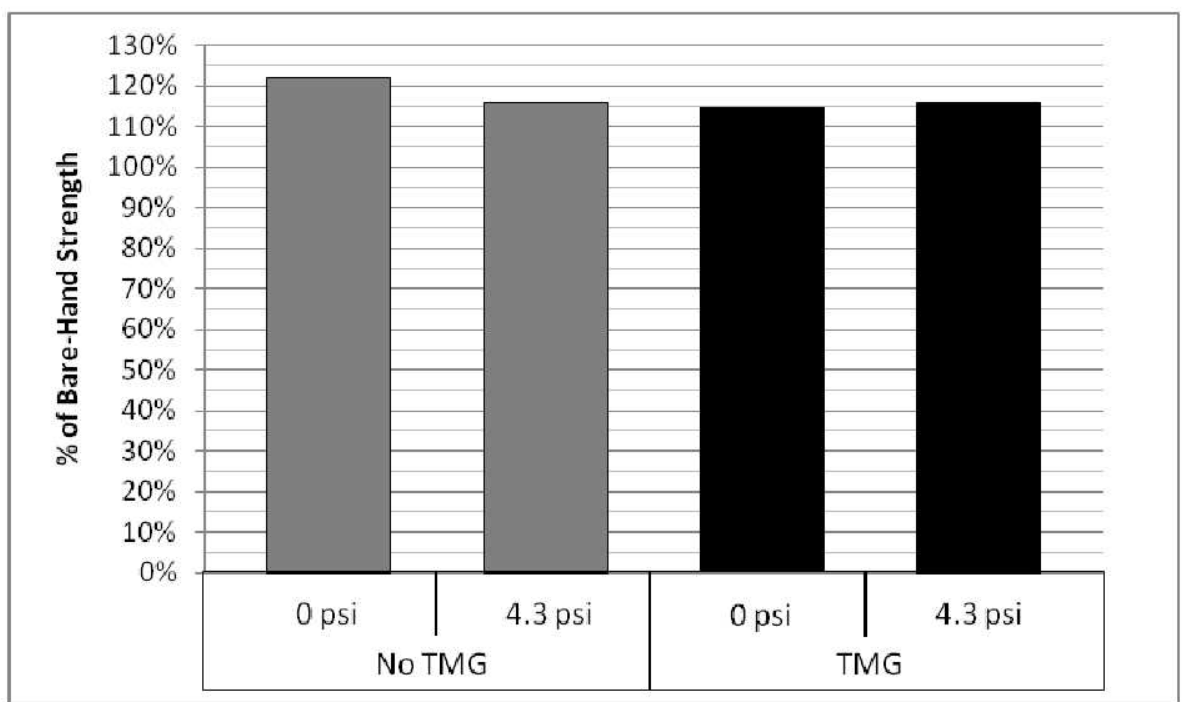

Figure 7. Pulp-2 Pinch Strength Results as a Percentage of Bare-Hand Strength

Unlike the other percentage of bare-hand strength results, the pulp-2 pinch strength performance increased while wearing the Phase VI Glove. All gloved configurations were above $110 \%$ of bare-hand strength. While pressurization slightly increased the strength ratio with a $\mathrm{TMG}$, pressurizing the glove without a TMG decreased the percentage to bare-hand by about $5 \%$.

\section{DISCUSSION}

To verify that the subject represented the population well, a comparison was made with existing literature. With the bare-hand grip strength ranging between $60 \mathrm{lbf}$ and $135 \mathrm{lbf}$, the expected astronaut population was represented well as an earlier study of astronaut grip strength reported a range of $65 \mathrm{lbf}-150 \mathrm{lbf}$ (Rajulu, 1993). For bare-hand lateral pinch strength, a previous study (Mathiowetz, 1984) reported an average range for the general population of $16 \mathrm{lbf}-25 \mathrm{lbf}$, which compares well with the range of this study being between $17 \mathrm{lbf}$ and $28 \mathrm{lbf}$. Pulp-2 pinch was also on-point with a literature range of $11 \mathrm{lbf}-17 \mathrm{lbf}$ (Mathiowetz, 1984) compared to the bare-hand range of $7 \mathrm{lbf}-18 \mathrm{lbf}$ found in this study.

Reduced applied hand strength due to wearing an unpressurized glove alone can significantly reduce performance. This was evident mostly in the grip strength evaluation. On average, subjects wearing an unpressurized glove without a TMG could produce only $55 \%$ of the force seen when bare-handed. Therefore, understanding how the glove can reduce certain hand strengths is of utmost importance when designing interfaces with a gloved crewmember whether the suit is pressurized or unpressurized.

Pressurizing the Phase VI Glove noticeably decreased hand grip strength, but had little to no affect on the lateral and pulp-2 pinch strengths. The cause of this 
difference is possibly due to the mechanics of the glove. When pressurized, hand postures like grip may require more force to overcome the natural shape of the glove, while the glove design may be more conducive to lateral and pulp-2 pinch postures. In other words, the subject is applying the same force as when barehanded, but the motion may be resisted or encouraged by the pressurized glove.

The TMG decreased the hand grip and pulp-2 pinch strengths, but had no affect on the lateral pinch strength. With more than a $10 \%$ decrease seen in barehand grip strength, designers of the next generation glove could greatly improve performance by looking for a novel solution in glove thermal and micrometeoroid protection. This could decrease fatigue and also prevent injuries associated with repeated tool manipulation.

\section{CONCLUSIONS}

While quantifying the characteristics of glove performance can be challenging at times, future designs will benefit by base lining existing hardware as this study did with the Phase VI Glove. The fluctuations in applied hand strength associated with pressurization and the TMG will also aid in the design of tools and other humanmachine interfaces. These types of studies are also useful across other industries that require a gloved hand to manipulate tools and interface with other hardware. Careful consideration should be taken of how hand strength is affected by a glove before assuming the hand will perform the same way as bare-handed.

Future work on gloved strength performance could involve glove box testing, fatigue studies, improved methods to quantify glove fit, and also incorporation of new technologies for data acquisition to get a better understanding of what is happening inside the glove (Hinman-Sweeney, 1994). The current EMU does not accommodate humans at the extremes of the anthropometric spectrum. For this reason, additional testing could be performed in a differential pressure glove box using the same methods as this grip and pinch strength evaluation. Smaller women and very large men who cannot be properly fitted with an EMU suit would be used as subjects. The results could then be compared to this study. Fatigue studies can be useful in assessing what types of motions and tools are best for longduration EVAs or highly repetitive tasks. Glove fit is an integral part of task performance and very difficult to quantify due to the natural subjectivity in human preferences. Although they are normally derived using questionnaires, metrics of glove fit would benefit if hand position inside the glove could be measured. New technologies such as internal pressure sensors could help with glove fit and also quantifying the force exerted by the subject on the glove, and not only the dynamometer or load cell. 


\section{REFERENCES}

Hinman-Sweeney, E.M., (1994), Extra-Vehicular Activity (EVA) Glove Evaluation Test Protocol. NASA Technical Memorandum, NASA TM 108442, Lyndon B. Johnson Space Center, Houston, Texas.

Mathiowetz, V., Kashman, N., Volland, G., Weber, K., Dowe, M., Rogers, S., (1984), Grip and Pinch Strengh: Normative Data for Adults. Occupational Therapy Program, University of Wisconsin-Milwaukee, Milwaukee, Wisconsin.

Rajulu, S.L., Bishu, R.R. (1993), A Comparison of Hand Grasp Breakaway Strengths and Bare-Handed Grip Strengths of the Astronauts, SML III Test Subjects, and the Subjects From the General Population. NASA Technical Paper 3286, Lyndon B. Johnson Space Center, Houston, Texas. 